\section{Lebensstilberatung mit lebenslanger Wirkung}

Patienten mit hohem KHK-Risiko regelmäBig zu gesunder Ernährung und Tabakabstinenz anzuhalten, lohnt sich, wie eine Studie aus Oslo bestätigt. In der 1972 begonnenen Studie waren Männer mit kardialen Risikofaktoren nach dem Zufallsprinzip einer Interventionsgruppe mit halbjährlicher Lebensstilberatung oder einer Kontrollgruppe ohne Beratung zugeteilt worden. Der Nutzen der Intervention zeigte sich nicht nur nach fünf Jahren in einer um $47 \%$ reduzierten Herzinfarktrate ( $2,7 \%$ vs. $5,7 \%)$. Noch nach 40 Jahren war die Herzinfarktmortalität niedriger als in der einstigen Kontrollgruppe.

J Intern Med 2016

\section{Keime aus Patientenhand}

Handhygiene ist eine wichtige Strategie, um die Verbreitung von Bakterien mit Mehrfachresistenzen einzudämmen. Dies gilt, so das Ergebnis einer US-Studie, auch für Patienten. Bei jedem vierten von 357 älteren Patienten, die in eine Rehaklinik aufgenommen wurden, stellten die Ärzte multiresistente Erreger auf den Händen fest. Während des Aufenthalts war sogar jeder Dritte mit den Problemkeimen kolonisiert. Getestet wurde auf Methicillin-resistenten Staphylococcus aureus, Vancomycin-resistente Enterokokken und (gegen Ceftazidim, Ciprofloxacin oder Imipenem) resistente gramnegative Bakterien.

JAMA Intern Med 2016; online 14. März 2016

\section{Schlaflos auf Station}

Mitarbeiter in den Kinderkliniken beobachten bei längeren Aufenthalten im Krankenhaus unter den Begleiteltern oft ein erhebliches Stresslevel. Deshalb wurde in einer Studie aus Großbritannien untersucht, wie gut Eltern in Kinderkliniken schlafen. Wie zu erwarten, berichteten diese über eine erheblich verminderte Schlafqualität und -dauer während des Aufenthalts. Dies trägt stark zur emotionalen Instabilität und zum erhöhten Stresslevel bei. Für Abhilfe könnten Veränderungen der Arbeitsabläufe während der Nacht sowie ein reduzierter Geräuschpegel sorgen. Dies könnte Eltern und Pflegepersonal das Leben auf Dauer einfacher machen.

Stickland A et al. Arch Dis Child 2016 Mar 24

\title{
Blutgruppe beeinflusst Thromboserisiko
}

Bereits frühere Studien ließen vermuten, dass Träger der Blutgruppen A, B oder AB stärker gefährdet sind, venöse oder kardiale Thromboembolien zu erleiden. Über die Ausprägung war bisher allerdings wenig bekannt. Nun erhärtet sich der Verdacht.

W issenschaftler um Senthil K Vasan, Stockholm, haben deshalb einen Datensatz mit über einer Million gesunder Blutspender aus Schweden und Dänemark ausgewertet und mit den entsprechenden Daten von Patientenregistern in Beziehung gesetzt. In einem Follow-up von 12,5 Jahren fanden sich 9.170 venöse Thromboembolien (VTE) und 24.653 kardiovaskuläre Ereignisse. Für Blutspender mit Blutgruppe A, B oder $\mathrm{AB}$ war das relative Risiko für tiefe Venenthrombosen und Lungenembolien im Vergleich zu 0-Blutgruppenträgern um fast das Doppelte erhöht (Inzidenzrate-Ratio, IRR: 1,92 und 1,80).

Ein besonders hohes Thromboserisiko im Vergleich zu 0-Blutgruppenträgern wiesen Personen mit Blutgruppe $\mathrm{AB}$ auf. Ebenfalls signifikant, aber geringer ausgeprägt, war das Risiko für Nicht0-Blutgruppenträger, ein kardiales Ereignis wie Herzinfarkt oder Schlaganfall zu erleiden (IRR: 1,10 und 1,07). Nach Ansicht von Vasan und Kollegen könnte es deshalb sinnvoll sein, die Blutgruppe in Thromboserisiko-Assessments bzw. in Prognose-Scores zu berücksichtigen. Immerhin sei die Bestimmung relativ einfach und werde vermutlich nicht von einer Akut-Phase-Reaktion beeinflusst.

(vsc)

Vasan, SK, Rostgaard, K, Majeed, A. et al. ABO Blood Group and Risk of Thromboembolic and Arterial Disease: A Study of 1.5 Million Blood Donors. Circulation. 2016 Apr 12;133(15):1449-57.

\section{Multiresistente Erreger im Pflegeheim}

Multiresistente Erreger (MRE) sind längst nicht mehr nur ein Problem von Kliniken. Auch Pflegeheime sind zunehmend betroffen. Ein Team vom Institut für Health Care Management der Universität Greifswald errechnete, welche Kosten Pflegeheimen entstehen, wenn Fälle von MRE auftreten.

$\mathrm{D}$ azu zählten die Forscher um Claudia Hübner dokumentierte Fälle von MRSA sowie multiresistente Gramnegative Bakterien, die gegen drei beziehungsweise vier Klassen von Antibiotika resistent sind (3-MRGN bzw. 4-MRGN). Als MRE-Fälle werteten die Forscher nicht nur Infizierte, sondern auch Patienten, die nachweislich mit Erregern aus den genannten Gruppen kolonisiert waren. An der Analyse nahmen sechs Pflegeeinrichtungen mit einer durchschnittlichen Anzahl von 95,2 Betten pro Heim teil. Im dreijährigen Auswertungszeitraum (2011 bis 2013) wurden insgesamt 71 Fälle mit positivem MRE-Carrier-Status registriert; auf jedes Heim kamen damit im Schnitt insgesamt 11,8 Fälle oder 3,9 Fälle pro Jahr. Die Falldauer (MRE-positive Tage) be- lief sich im Mittel auf 163,3 Tage. Nach Hübner et al. schlug damit ein einzelner MRE-Fall mit insgesamt 12.448,36 Euro zu Buche. Der Löwenanteil entfiel dabei auf die zusätzliche Arbeitslast (7.177,04 Euro) und die Isolationsmaterialien (4.033,51 Euro).

Die Wissenschaftler warnen, dass die Chance, MRSA in Pflegeheimen zu identifizieren, deutlich geringer sei als in Kliniken; dies liege daran, dass in den Heimen in der Regel keine spezifische mikrobiologische Diagnostik zum Einsatz komme. Tatsächlich müsse man wohl von einer deutlich höheren Erregerlast ausgehen als der hier berichteten. (eo)

Huebner C, Roggelin M, Flessa S. Economic burden of multidrug-resistant bacteria in nursing homes in Germany: a cost analysis based on empirical data. BMJ Open 2016; 6: e008458 